\title{
First-principles Study on Surface Stability of Tantalum Carbides
}

\author{
Wen-Li Yan ${ }^{\mathrm{a}, \mathrm{b}}$, Michael Sygnatowicz ${ }^{\mathrm{b}}$, Guang-Hong Lua ${ }^{\mathrm{a}}$, Feng Liu ${ }^{\mathrm{b}, *}$, Dinesh K. Shetty ${ }^{\mathrm{b}}$ \\ ${ }^{a}$ Department of Physics, Beihang University, Beijing 100191, P. R. China \\ ${ }^{\mathrm{b}}$ Department of Materials Science and Engineering, University of Utah, Salt Lake City, Utah 84112, \\ United States
}

\begin{abstract}
Using first-principles method, surface energies of crystal planes of different tantalum carbide phases have been calculated. Quantum size effects are shown to possibly play a considerable role in determining accurate surface energies of these metallic films, which have been neglected in previous works. The $\gamma$-TaC phase has a more stable $\left(\begin{array}{lll}0 & 0 & 1\end{array}\right)$ surface than the close-packed $\left(\begin{array}{lll}1 & 1 & 1\end{array}\right)$ surface. In the $\alpha-\mathrm{Ta}_{2} \mathrm{C}$ phase, $\left(\begin{array}{lll}0 & 0 & 1\end{array}\right)$ surface with only Ta termination is more stable than that of mixed Ta-C termination, because the metallic bonds between Ta atoms are weaker than the Ta-C covalent bonds. The same is true for the $\zeta-\mathrm{Ta}_{4} \mathrm{C}_{3}$ phase. The introduction of structural vacancies in the $\zeta-\mathrm{Ta}_{4} \mathrm{C}_{3-\mathrm{x}}$ phase creates more direct Ta metallic bonds, making the Ta terminated surfaces even more stable. This is consistent with the experimental observations of cleavage of the basal planes, lamellae bridging of cracks, and the high fracture toughness of $\zeta-\mathrm{Ta}_{4} \mathrm{C}_{3-\mathrm{x}}$.
\end{abstract}

Keywords: First principles, Surface energy, Transition metal carbides

\footnotetext{
* Corresponding author.

E-mail address: fliu@eng.utah.edu (F. Liu)
} 


\section{Introduction}

Tantalum carbides offer attractive properties ranging from high melting points and thermal stability, high chemical resistance, and high hardness and strength [1-3], rendering them useful in such applications as rocket motor nozzle liners [4], refractory crucibles for containing corrosive liquids and melts [5,6], and as grain-growth inhibitors in cermets for cutting tools, and so on. Besides those properties pertaining to typical covalent crystals, the mixed covalent-metallic bonds in tantalum carbides also provide them with good thermal and electrical conductivity $[1,2]$ as well as catalytic activities [7].

The Ta-C binary system offers three major carbides with different crystal structures in different ranges of $\mathrm{C} / \mathrm{Ta}$ atomic ratio [8]. All of them can be represented by the empirical chemical formula, $\mathrm{TaC}_{\mathrm{x}}$. The $\gamma-\mathrm{TaC}$ phase has a rock salt $\mathrm{B} 1$ structure with a face-centered-cubic stacking sequence and is stable as a single phase over the composition range, $0.82<\mathrm{x}<1.0$, at low temperatures $(\mathrm{T} \sim 500 \mathrm{~K})$. The $\alpha-\mathrm{Ta}_{2} \mathrm{C}$ phase has an anti-cadmium iodide $\mathrm{C} 6$ structure with a hexagonal-close-packed Ta stacking sequence and is stable as a single phase in the composition range, $0.44<\mathrm{x}<0.5$. The $\zeta-\mathrm{Ta}_{4} \mathrm{C}_{3}$ phase has a trigonal lattice, and the Ta stacking sequence is composed of alternating face-centered-cubic and hexagonal-close-packed stacking blocks interrupted by stacking faults and missing carbon layers [8]. It is stable in the composition range, $0.65<\mathrm{x}<0.68[8]$. Because of their high non-stoichiometry, the three tantalum carbide phases are represented as $\gamma-\mathrm{TaC}_{\mathrm{y}}, \alpha-\mathrm{Ta}_{2} \mathrm{C}_{\mathrm{y}}$, and $\zeta-\mathrm{Ta}_{4} \mathrm{C}_{3-\mathrm{x}}$ respectively [8,9]. The amount of structural vacancies can reach tens of atomic percentage [8], affecting crystal structures [10] and various mechanical and electrical properties [11] of these phases. According to recent experimental studies, tantalum carbides containing high weight fractions of the $\zeta-\mathrm{Ta}_{4} \mathrm{C}_{3-\mathrm{x}}$ phase have shown high fracture toughness and increasing crack-growth-resistance behavior [12-15]. The $\zeta-\mathrm{Ta}_{4} \mathrm{C}_{3-\mathrm{x}}$ 
phase grains were highly anisotropic measuring $\sim 20-30 \mu \mathrm{m}$ in length and $\sim 0.97-1.37 \mu \mathrm{m}$ in thickness [13]. Optical and scanning electron microscope examinations of the specimen lamellae under single-edge pre-cracked beam (SEPB) test suggest a crack-face bridging toughening mechanism for the $\zeta-\mathrm{Ta}_{4} \mathrm{C}_{3-\mathrm{x}}$ phase. The lamellae are formed by cleavage of the basal planes which bridge the cracks and hinder their propagation [15]. Since the relative ease of cleavage of the close-packed basal planes should correlate with the respective surface energies, the surface energies were calculated for the crystal planes of the three major tantalum carbide phases using the first-principles method.

For metallic materials such as tantalum carbides, the electron Fermi wavelength is short (in the range of 3 5 $\AA$ ) compared to semiconductors and insulators [16]. Consequently, pronounced quantum size effect (QSE) induced by electron quantum confinement is expected in their lowdimensional nanostructures [17], which poses substantial influences on electron charge density, work function, surface energy, surface stress, dielectric properties, and so on [18-19]. This also calls for a careful calculation of surface energies using the common thin slab method, consisting of adequate number of layers, because the surface energy may display an oscillating thickness dependency [17].

In this paper, first-principles study has been made on the surface energies of the three stoichiometric phases, $\gamma-\mathrm{TaC}, \alpha-\mathrm{Ta}_{2} \mathrm{C}$, and $\zeta-\mathrm{Ta}_{4} \mathrm{C}_{3}$, and the high-fracture-toughness nonstoichiometric $\zeta-\mathrm{Ta}_{4} \mathrm{C}_{3-\mathrm{x}}$ phase. Especially, QSEs are carefully considered in determining accurate surface energies of these metallic films, which have been neglected in previous studies [20-21]. The Ta terminated surface formed by the basal planes of $\zeta-\mathrm{Ta}_{4} \mathrm{C}_{3-\mathrm{x}}$ phase is found to be more stable than that of $\zeta-\mathrm{Ta}_{4} \mathrm{C}_{3}$, due to weaker metallic bonds between Ta atoms introduced by the structural vacancies at $\mathrm{C}$ sites. Our calculation results are consistent with experimental 
observations that the $\zeta-\mathrm{Ta}_{4} \mathrm{C}_{3-\mathrm{x}}$ phase is more likely to form lamellar structure under loading, thus leading to the bridging mechanism that produces high fracture toughness [15].

\section{Calculation method}

All first principles calculations were performed using Vienna Ab-initio Simulation Package (VASP) [22] within the density functional theory. The interactions between the electrons and ions were described using the projector augmented wave (PAW) method [23,24]. The scheme of Perdew, Burke, and Ernzerhof (PBE) [25] within the general gradient approximation (GGA) was used for the electron exchange-correlation functional. The PAW-PBE potentials were obtained from the VASP package, using the valence electron configurations of $2 s^{2} 2 p^{2}$ and $5 p^{6} 6 s^{2} 5 d^{3}$ for $\mathrm{C}$ and Ta, respectively. The Brillouin zone was integrated using the Methfessel-Paxton smearing method [26] of first order with the smearing parameter $\sigma$ of $0.1 \mathrm{eV}$, except for calculations of very accurate total energy without relaxation, where the tetrahedron method with Blöchl corrections [27] was used. All calculations used a plane-wave kinetic energy cutoff of $500 \mathrm{eV}$ to achieve good convergence for stress tensor. The $\Gamma$-centered Monkhorst-Pack k-point grid [28] was used, and the k-point sampling (details listed in Table 1) with a resolution of around $2 \pi \times$ $0.02 \AA^{-1}$ converges the total energy to within $1 \mathrm{meV}$.

For bulk calculations of $\mathrm{TaC}, \mathrm{Ta}_{4} \mathrm{C}_{3}$, and $\mathrm{Ta}_{2} \mathrm{C}$, primitive cells were used in which the numbers of Ta atoms and $\mathrm{C}$ atoms are the same as those in the chemical formulae respectively (Fig. 1). The cell volume was determined by fitting a computed energy-volume curve to the Birch-Murnaghan equation of state [29], and the atomic positions and cell structure were optimized with an ionic force threshold of $0.001 \mathrm{eV} / \AA$. 
For the surface calculations, the supercell model is constructed with a slab and vacuum, which were acquired by cleaving the bulk equilibrium structures calculated above. The surface indices are with respect to the conventional unit cell, i.e. cubic for $\mathrm{TaC}$ while trigonal for $\mathrm{Ta}_{4} \mathrm{C}_{3}$, and $\mathrm{Ta}_{2} \mathrm{C}$. The vacuum layer thickness is set to be $20 \AA$, large enough to suppress spurious interaction between periodic images. The atomic positions were allowed to relax within the fixed cell volume and shape to realize possible surface rumpling in the ground state [30], and the ionic force threshold was also set to be $0.001 \mathrm{eV} / \AA ̊$. For asymmetric surfaces with different terminations, dipole corrections are included.

\section{Results and discussion}

\subsection{Bulk Crystal Structure and Mechanical Properties}

As a benchmark study, the bulk structures and elastic moduli of $\mathrm{TaC}, \mathrm{Ta}_{4} \mathrm{C}_{3}$, and $\mathrm{Ta}_{2} \mathrm{C}$ are calculated and listed in Table 1 . The calculated results of lattice constants are always slightly larger than the experimental values $[8,31,32]$, but within a reasonable range, which is originated from the GGA exchange-correlation functional we have chosen in our study. 
(a)
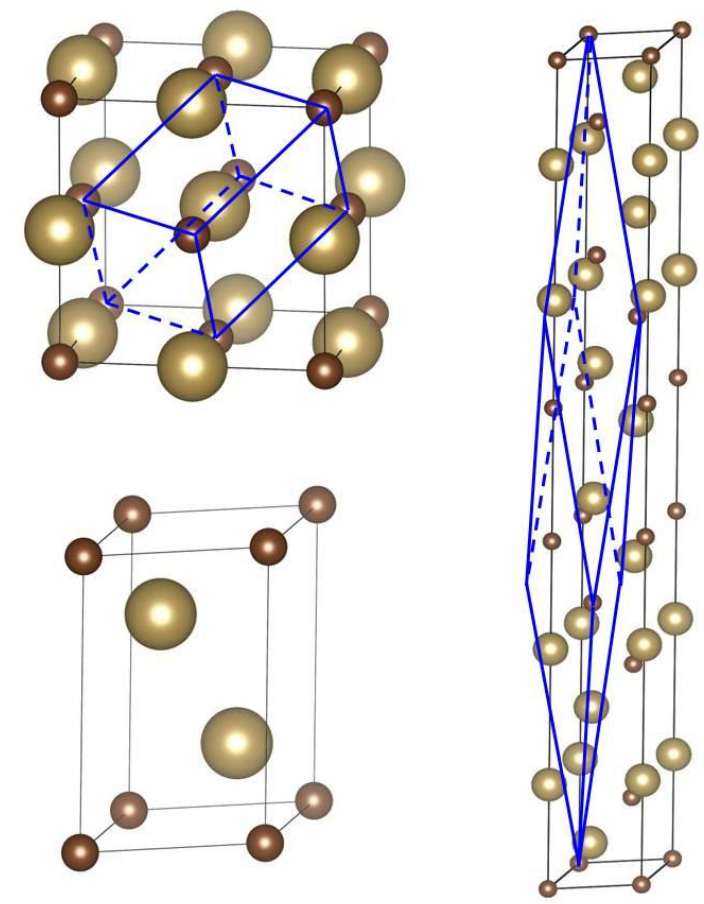

(c)

(b)

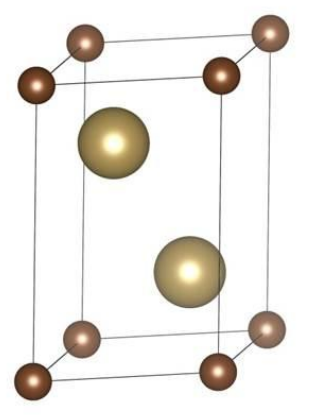

Fig. 1. Unit cell structure of (a) $\mathrm{TaC}$, (b) $\mathrm{Ta}_{2} \mathrm{C}$, and (c) $\mathrm{Ta}_{4} \mathrm{C}_{3}$, presented using VESTA [33]. The large yellow sphere represents Ta atom, and the small brown sphere represents $\mathrm{C}$ atom. The three diagrams are not in the same scale. For $\mathrm{TaC}_{\mathrm{C}}$ and $\mathrm{Ta}_{4} \mathrm{C}_{3}$, the blue grids show the primitive cells.

The bulk retains linear elasticity under small strains, and there exists linear relationship between stress and strain according to the Hook's law. By designing a series of small strains along different directions and calculating the corresponding stress tensor, all the elastic stiffness constants $C_{i j}$ for the bulk phases can be calculated. Applying the Voigt-Reuss-Hill approximation [32], the bulk modulus $B$ and shear modulus $G$ can be calculated, in which Voigt's and Reuss's schemes represent the upper and lower bounds, and the Hill's scheme takes average of the two:

$$
\begin{aligned}
& B_{V}=\frac{1}{9}\left(C_{11}+C_{22}+C_{33}\right)+\frac{2}{9}\left(C_{12}+C_{13}+C_{23}\right) \\
& B_{R}=\frac{1}{\left(S_{11}+S_{22}+S_{33}\right)+2\left(S_{12}+S_{13}+S_{23}\right)}
\end{aligned}
$$


and

$$
\begin{aligned}
& G_{V}=\frac{1}{15}\left(C_{11}+C_{22}+C_{33}-C_{12}-C_{13}-C_{23}\right)+\frac{1}{5}\left(C_{44}+C_{55}+C_{66}\right) \\
& G_{R}=\frac{15}{4\left(S_{11}+S_{22}+S_{33}\right)-4\left(S_{12}+S_{13}+S_{23}\right)+3\left(S_{44}+S_{55}+S_{66}\right)},
\end{aligned}
$$

in which $S_{i j}$ represents the elastic compliance constants consisting of the compliance tensor which is the inverse of the stiffness tensor. The Young's modulus $E$ and Poisson's ratio $v$ can then be derived as,

$$
E=\frac{9 B G}{3 B+G}, v=\frac{3 B-2 G}{2(3 B+G)} .
$$

Our calculated elastic moduli (Table 1) are consistent with available experimental [35] and other calculation results $[36,37]$. Comparing among the three phases, we can see that with decreasing $\mathrm{C} / \mathrm{Ta}$ ratio, the elastic modulus also decreases.

\section{Table 1}

Crystal structure and elastic moduli of bulk $\mathrm{TaC}, \mathrm{Ta}_{4} \mathrm{C}_{3}$, and $\mathrm{Ta}_{2} \mathrm{C}$. Lattice constants are with respect to the conventional unit cell, and for $\mathrm{Ta}_{4} \mathrm{C}_{3}$ and $\mathrm{Ta}_{2} \mathrm{C}$, the lattice constant a is followed by c. Experimental values of lattice constants and elastic moduli are also listed in italics for reference.

\begin{tabular}{cccc}
\hline & $\mathrm{TaC}$ & $\mathrm{Ta}_{4} \mathrm{C}_{3}$ & $\mathrm{Ta} \mathrm{C}$ \\
\hline Space Group (No.) & $F m \overline{3} m(225)$ & $R \overline{3} m(166)$ & $P \overline{3} m 1(164)$ \\
k-points mesh & $21 * 21 * 21$ & $21 * 21 * 21$ & $21 * 21 * 11$ \\
Lattice Constant $(\AA)$ & 4.48 & $3.15,30.42$ & $3.12,4.96$ \\
\hline
\end{tabular}




\begin{tabular}{|c|c|c|c|}
\hline & $4.4570[31]$ & $3.1216,30.058[8]$ & $3.1030,4.9378[32]$ \\
\hline Atom positions & $\begin{array}{l}\mathrm{C}(4 a)(0,0,0) \\
\mathrm{Ta}(4 b)(0.5,0.5,0.5)\end{array}$ & $\begin{array}{l}\mathrm{C}(3 a)(0,0,0) \\
\mathrm{C}(6 c)(0,0,0.4196) \\
\mathrm{Ta}(6 c)(0,0,0.1259) \\
\mathrm{Ta}(6 c)(0,0,0.2895)\end{array}$ & $\begin{array}{l}\mathrm{C}(1 a)(0,0,0) \\
\mathrm{Ta}(2 d)(0.3333, \\
0.6667,0.2529)\end{array}$ \\
\hline $\mathrm{B}(\mathrm{GPa})$ & $\begin{array}{c}325 \\
332 \pm 39[35]\end{array}$ & 287 & 259 \\
\hline $\mathrm{G}(\mathrm{GPa})$ & $\begin{array}{c}212 \\
234 \pm 27[35]\end{array}$ & 181 & 142 \\
\hline $\mathrm{E}(\mathrm{GPa})$ & $\begin{array}{c}522 \\
567 \pm 68[35]\end{array}$ & 448 & 360 \\
\hline$v$ & $\begin{array}{c}0.232 \\
0.215 \pm 0.020[35]\end{array}$ & 0.240 & 0.268 \\
\hline
\end{tabular}

\subsection{Surface Energy}

With the equilibrium bulk structure at hand, we can directly cleave the bulk and make slabs of fixed volume and shape, since the bulk layers of the slab should keep the structure of bulk in a reasonable surface model no matter how the surface layers are reconstructed or rumpled. The specific surface energy can be calculated using the following equation,

$$
E_{\text {surf }}=\frac{E_{\text {slab }}-n E_{\text {bulk }}}{2 A}
$$

where $E_{\text {slab }}$ is the total energy of the slab containing specific number of atomic layers, $E_{b u l k}$ is the total energy of the bulk unit cell, and the multiplier $n$ is to equalize the total number of atoms in the slab with that in the bulk. $A$ represents the surface area, and the factor 2 is used because the slab has two surfaces. Smaller surface energy indicates surfaces of higher stability. 
The most common method to calculate the surface energy is conducting two separate calculations to determine the slab energy and the bulk energy, respectively. However, due to different cell size and k-point sampling, unavoidable systematic error would occur [38], and the surface energies calculated using different layers of slab can diverge. Therefore, a slab method is proposed to extract convergent surface energies from slab calculations [39], in which slab energies are fitted in relative to the number of slab layers and the slope is taken as the bulk energy.

Another important reason for considering a series of slab thicknesses resides in the QSE of metallic films. When the thickness of a metal film is reduced to the range of the electron Fermi wavelength, quantum confinement becomes prominent to form discrete quantum well states. As a result, surface energies will oscillate until the thickness of the slab becomes large. Figure 2 shows the surface energy and surface stress plot with changing number of monolayers for the $\mathrm{TaC}\left(\begin{array}{lll}0 & 0\end{array}\right)$ surface. The inset shows the linear fitting of slab total energy with respect to number of monolayers when the layer number is larger than 10. The bulk energy is fitted as the slope with the standard error of $0.46 \mathrm{meV}$. We can see that the surface energy varies by $2 \mathrm{meV} / \AA^{2}$ per layer even when the number of monolayers reaches 20 . The surface stress also shows the same beating pattern. From this plot, the converged surface energy is determined as $95 \mathrm{meV} / \AA^{2}(1.51$ $\mathrm{J} / \mathrm{m}^{2}$ ). The result is much smaller than $2.99 \mathrm{~J} / \mathrm{m}^{2}$ reported in ref. 20, since the ignorance of surface relaxation in that reference results in a large error for severely rumpled $\mathrm{TaC}\left(\begin{array}{lll}0 & 0 & 1\end{array}\right)$ surface $[21,30]$. 


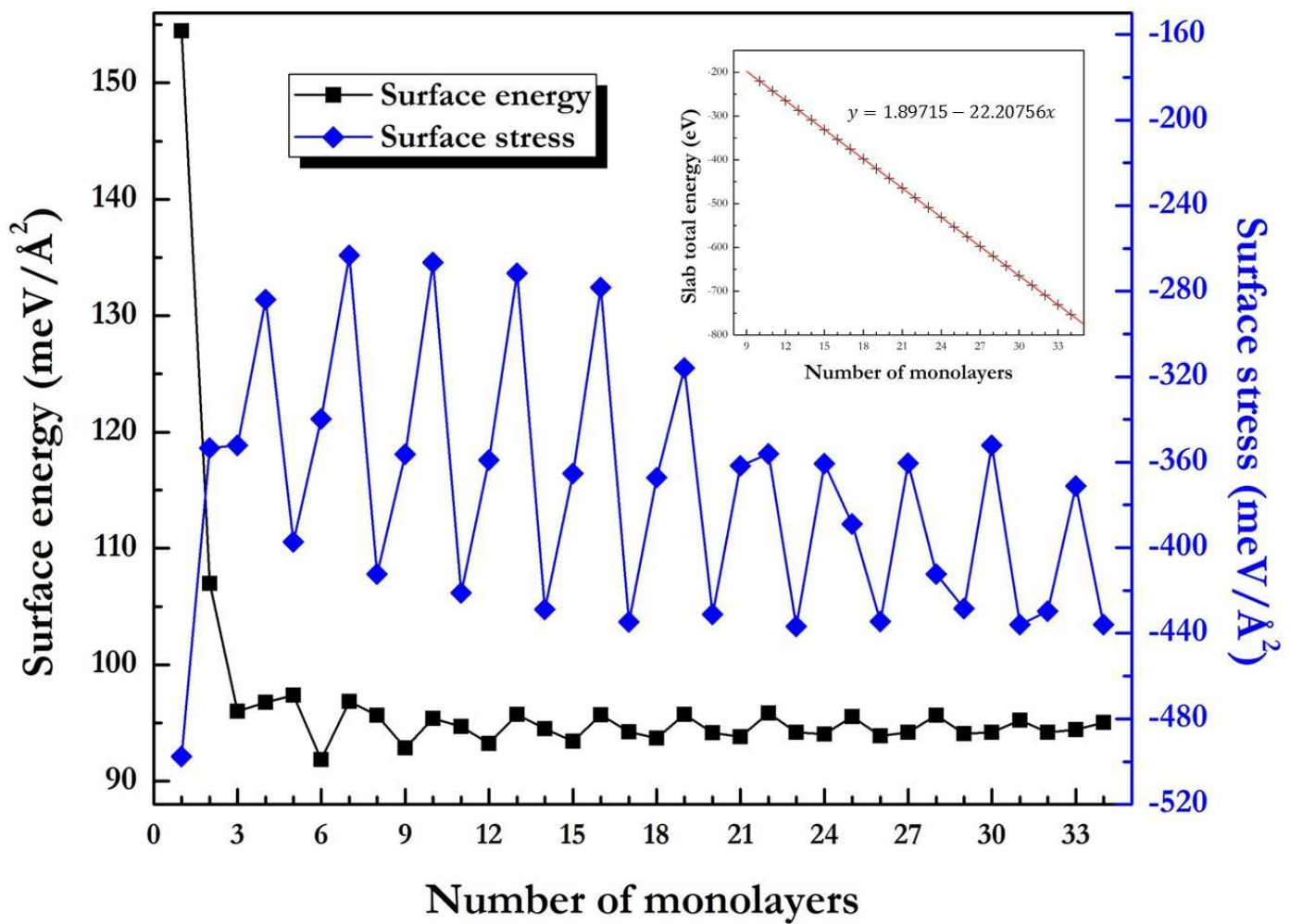

Fig. 2. Surface energy and surface stress of $\mathrm{TaC}\left(\begin{array}{lll}0 & 0 & 1\end{array}\right)$ surface as a function of the number of monolayers. The inset is the linear fitting of slab total energy with respect to number of monolayers.

To estimate the strength of QSE in TaC films, we have calculated the band structure and density of states of bulk $\mathrm{TaC}$, as shown in Fig. 3, which indicate that $\mathrm{TaC}$ is a strong metal with the Fermi level lying in the mix of Ta $5 d$ bands and C $2 p$ bands. Accordingly, accounting three $5 d$ electrons of Ta and two $2 p$ electrons of $\mathrm{C}$ as the "free" valence electrons and using Sommerfeld's free-electron-gas model, we estimated the Fermi wavelength of TaC to be $\lambda_{F} \sim$ $3.35 \AA$ A. Given the interlayer spacing of $\mathrm{TaC}\left(\begin{array}{lll}0 & 0 & 1\end{array}\right)$ film to be $d_{0} \sim 2.24 \AA$, we have $d_{0}: \frac{\lambda_{F}}{2} \approx 4: 3$. This suggests a strong QSE in $\mathrm{TaC}\left(\begin{array}{lll}0 & 0 & 1\end{array}\right)$ film with its surface properties 
oscillating in a period of three layers [17-19,40], in good agreement with our direct DFT calculations as shown in Fig. 2.

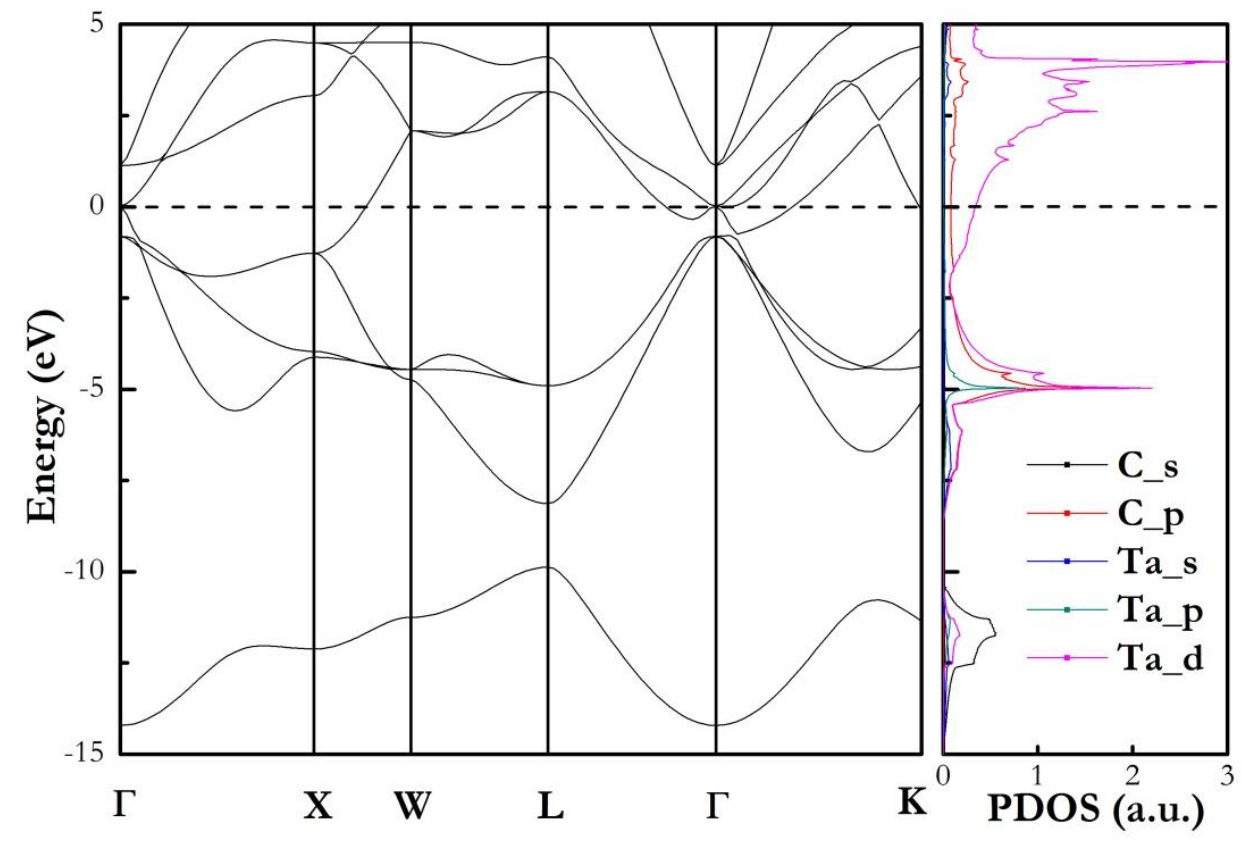

Fig. 3. Band structure along several high symmetry directions and partial density of states (PDOS) of bulk TaC. The energy reference is set to the Fermi level (dashed horizontal line).

Using the same method, we have also determined the surface energy of TaC $\left(\begin{array}{lll}1 & 1 & 1\end{array}\right)$ to be $0.255 \mathrm{eV} / \AA^{2}\left(4.08 \mathrm{~J} / \mathrm{m}^{2}\right)$. Though Ta atoms form a face-centered-cubic stacking sequence and the (1 11 1) planes are closely packed, the number of broken bonds is not the fewest with $\mathrm{C}$ sublattice interweaving, which explains why the TaC $\left(\begin{array}{lll}1 & 1 & 1\end{array}\right)$ surface has higher surface energy than the $\left(\begin{array}{ll}0 & 0\end{array}\right.$ 1) surface, contrary to the situations in fcc metals [41].

The $\mathrm{Ta}_{2} \mathrm{C}\left(\begin{array}{lll}0 & 0 & 1\end{array}\right)$ basal planes have two kinds of environments: one consists of two adjacent Ta-Ta planes, while the other consists of neighboring Ta-C planes (Fig. 1(b)). Therefore, we calculated $\mathrm{Ta}_{2} \mathrm{C}\left(\begin{array}{lll}0 & 0 & 1\end{array}\right)$ surfaces of two terminations. The Ta terminated surface has a surface 
energy of $0.181 \mathrm{eV} / \AA^{2}\left(2.90 \mathrm{~J} / \mathrm{m}^{2}\right)$ and the surface with mixed Ta-C termination has a surface energy of $0.314 \mathrm{eV} / \AA^{2}\left(5.04 \mathrm{~J} / \mathrm{m}^{2}\right)$. The latter has a higher surface energy and is less stable because breaking Ta-C covalent bond needs more energy than breaking Ta-Ta metallic "bonds" (interactions).

\subsection{Effect of Structural Vacancies in the $\zeta-\mathrm{Ta}_{4} \mathrm{C}_{3-x}$ Phase}

The $\left(\begin{array}{lll}0 & 0 & 1\end{array}\right)$ surfaces of the $\zeta$ phase are more complicated, since surfaces of different terminations and different cleaving positions must be considered. Figure 3(a) illustrates four nonequivalent surface cleaving methods for $\mathrm{Ta}_{4} \mathrm{C}_{3}$. Besides one Ta terminated surface, there are three kinds of surfaces with mixed Ta-C termination, and we denoted them as Ta-C 1, Ta-C 2, and Ta-C 3. The calculated surface energies and corresponding bonding energies are listed in Table 2. We can see that metallic bonds between Ta atoms are weaker compared to the Ta-C covalent bond, making the Ta terminated surface to be the most stable one.

\section{Table 2}

Surface energies and corresponding bonding energies of different surfaces.

\begin{tabular}{|l|c|c|c|c|}
\hline Surface type & Ta-Ta & Ta-C 1 & Ta-C 2 & Ta-C 3 \\
\hline Bonding energy (eV) & 0.92 & 1.91 & 1.48 & 1.58 \\
\hline Surface energy $\left(\mathrm{eV} / \AA^{2}\right)$ & 0.161 & 0.334 & 0.258 & 0.276 \\
\hline Surface energy $\left(\mathrm{J} / \mathrm{m}^{2}\right)$ & 2.58 & 5.35 & 4.14 & 4.42 \\
\hline
\end{tabular}

To model the $\mathrm{C}$ deficient non-stoichiometric $\zeta-\mathrm{Ta}_{4} \mathrm{C}_{3-\mathrm{x}}$ phase, we need to first identify a reasonable and realistic $\mathrm{x}$ value or $\mathrm{C} / \mathrm{Ta}$ ratio. Experiments found that the $\zeta-\mathrm{Ta}_{4} \mathrm{C}_{3-\mathrm{x}}$ phase is stable over the temperature range of $300 \sim 2400 \mathrm{~K}$ with a homogeneity interval from $\mathrm{TaC}_{0.65}$ to 
$\mathrm{TaC}_{0.68}[8]$. No matter what the ratio is, the positions $(6 c)$ are always occupied by $\mathrm{C}$ atoms, while the positions (3a) are only partially occupied [8]. Therefore we focus on a $\zeta-\mathrm{TaC}_{0.67}$ phase with uniform structural vacancy distribution for calculation convenience, in which two thirds of $(3 a)$ positions in the trigonal unit cell are occupied by $\mathrm{C}$ atoms while the other one thirds of $(3 a)$ positions are vacant or occupied by structural vacancies (Fig. 3(b)). The $\zeta$ phase with the same $\mathrm{C} / \mathrm{Ta}$ ratio as that of our model has also been chosen to study in experiments [8].

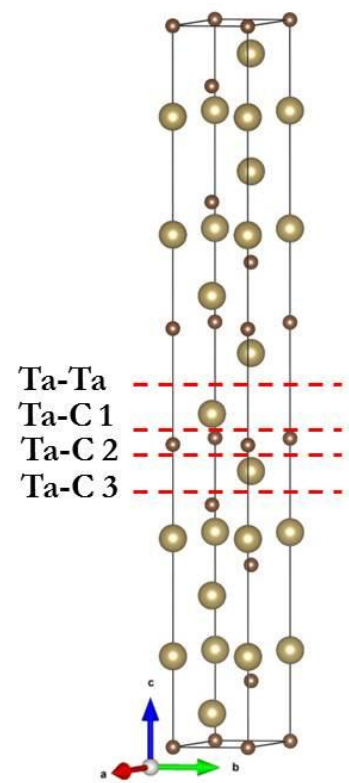

(a)

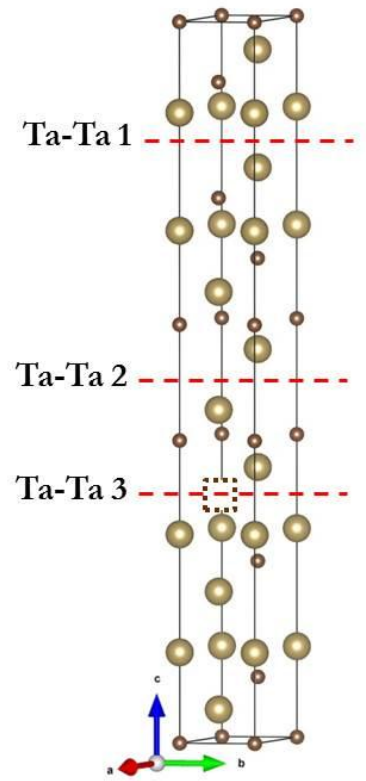

(b)

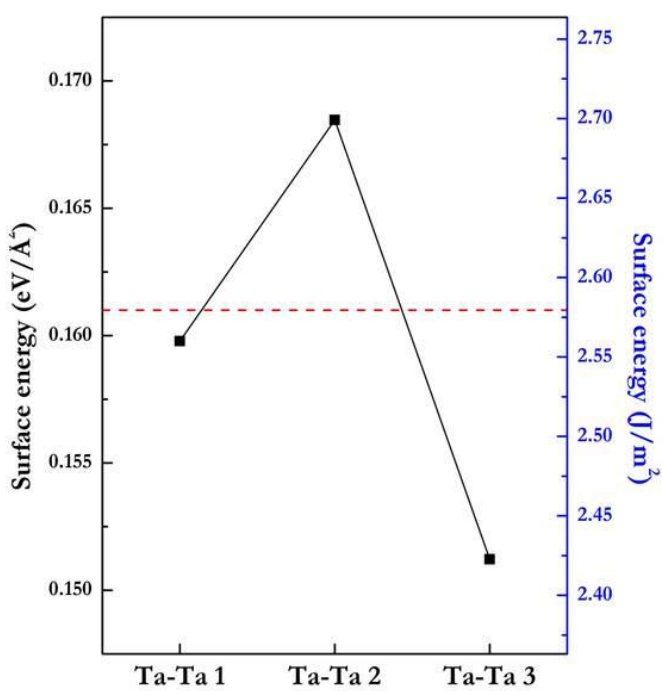

(c)

Fig. 3. (a) Four non-equivalent surface cleaving methods for $\zeta-\mathrm{Ta}_{4} \mathrm{C}_{3}$; (b) Three Ta terminated surface cleaving methods for $\zeta-\mathrm{Ta}_{4} \mathrm{C}_{3-\mathrm{x}}$; (c) Surface energies of the three Ta terminated surfaces of $\zeta-\mathrm{Ta}_{4} \mathrm{C}_{3-\mathrm{x}}$, with the red dashed line of surface energy for the Ta terminated surface in $\zeta-\mathrm{Ta}_{4} \mathrm{C}_{3}$ for reference.

With the introduced structural vacancy at C $(3 a)$ sites, two more kinds of Ta terminated surfaces can be formed compared to the stoichiometric $\mathrm{Ta}_{4} \mathrm{C}_{3}$ phase. Figure $3(\mathrm{c})$ shows the 
surface energies for all three Ta terminated surfaces, in comparison with the original surface energy in the stoichiometric phase. We can see that more stable Ta terminated surfaces can be formed with the structural vacancy present. Since more Ta-Ta direct metallic bonds are created with specific $\mathrm{C}$ layers missing in the non-stoichiometric phase, the material becomes easier to form lamellar structure, consistent with experimental observations [15].

\section{Conclusion}

The tantalum carbide phases can be perceived as progressive filling of $\mathrm{C}$ atoms at octahedral interstitial sites of Ta crystallographic lattice along (lll 111$)$ planes and the corresponding shifts of Ta planes and symmetry rearrangement [42], in which $\mathrm{Ta}_{4} \mathrm{C}_{3}$ was formed by removal of every fourth $\mathrm{C}$ plane in $\mathrm{TaC}$, and $\mathrm{Ta}_{2} \mathrm{C}$ was formed by continual removal of every third $\mathrm{C}$ plane in $\mathrm{Ta}_{4} \mathrm{C}_{3}$. The non-stoichiometric phases are represented by missing $\mathrm{C}$ atoms and thus less Ta-C bonds compared to the corresponding stoichiometric phases. From the point view of bond breaking in the process of surface formation, different surface energies can be understood by different bonding energy. The experimental high fracture toughness $\zeta-\mathrm{Ta}_{4} \mathrm{C}_{3-\mathrm{x}}$ phase is showed to have a lowered surface energy with introduced structural vacancies, which facilitates an easier lamellar structure formation and thus stronger crack-face bridging mechanism [15] sustaining the high facture toughness. In realistic experimental specimens, the expected higher local concentrations of structural vacancies in the $\zeta-\mathrm{Ta}_{4} \mathrm{C}_{3-\mathrm{x}}$ phase may lower the surface energy further and increase the structural anisotropy, which requires further investigations. In our calculations of surface energy, we found that the QSE possibly needs to be carefully considered, as the surface energy oscillates with number of monolayers in a wide range of layer thickness. 


\section{Acknowledgment}

W.-L. Yan and F. Liu acknowledge support by DOE-BES program (Grant No. DEFG0204ER46148). W.-L Yan is thankful for the support from China Scholarship Council. We thank the CHPC at the University of Utah for providing computing resources.

\section{References}

[1] H.O. Pierson, Handbook of Refractory Carbides \& Nitrides, Noyes Pubs., Westwood, NJ, 1996.

[2] A. Krajewski, L. D’Alessio, G. De Maria, Cryst. Res.Technol. 33 (1998) 341.

[3] N. Ahlén, M. Johnsson, M. Nygren, Thermochimica Acta, 336 (1999) 111.

[4] A. Metcalfe and N. B. Elsner, US Patent Application Publication No. US/2006/0144037 A1 (2006)

[5] J. Veligdan, D. Branch, P.E. Vanier, R.E. Barletta, Mater. Res. Soc. Symp. Proc. 285 (1992) 575.

[6] J. A. H. de Pruneda, US Patent No. 5,383,981 (1995).

[7] J.G. Choi, Appl. Catal. A 184 (1999) 189.

[8] A.I. Gusev, A.S. Kurlov, V.N. Lipatnikov, J. Solid State Chem. 180 (2007) 3234.

[9] A.I. Gusev, A.A. Rempel, A.J. Magerl, Disorder and Order in Strongly

Nonstoichiometric Compounds, Springer, 2001.

[10] D. Rowcliffe, G. Thomas, Mater. Sci. Eng. 18 (1975) 231.

[11] A.I. Gusev, JETP Lett. 90 (2009) 191. 
[12] K. Hackett, S. Verhoef, R.A. Cutler, and D.K. Shetty, J. Am. Ceram. Soc. 92 (2009) 2404.

[13] L. Liu, F. Ye, Y. Zhou, Z. Zhang, J. Am. Ceram. Soc. 93 (2010) 2945.

[14] M. Sygnatowicz, R.A. Cutler, D.K. Shetty, J. Am. Ceram. Soc. 97 (2014) 3826.

[15] M. Sygnatowicz, R.A. Cutler, D.K. Shetty, J. Am. Ceram. Soc. 98 (2015) 2601.

[16] N.W. Ashcroft, N.D. Mermin, Solid State Physics, Thomson Learning, New York (1976)

[17] F.K. Schulte, Surf. Sci. 55 (1976) 428.

[18] M. Liu, Y. Han, L. Tang, J.-F. Jia, Q.-K. Xue, F. Liu, Phys. Rev. B 86 (2012) 125427.

[19] W. Ming, S. Blair, F. Liu, J. Phys. Condens. Matter 26 (2014) 505302.

[20] H.W. Hugosson, O. Eriksson, U. Jansson, A.V. Ruban, P. Souvatzis, I.A. Abrikosov, Surf. Sci. 557 (2004) 243.

[21] F. Viñes, C. Sousa, P. Liu, J.A. Rodriguez, F. Illas, J. Chem. Phys. 122 (2005) 174709.

[22] G. Kresse, J. Furthmüller, Phys. Rev. B 54 (1996) 11169.

[23] P.E. Blöchl, Phys. Rev. B 50 (1994) 17953.

[24] G. Kresse, J. Joubert, Phys. Rev. B 59 (1999) 1758.

[25] J.P. Perdew, K. Burke, M. Ernzerhof, Phys. Rev. Lett. 77, 3865 (1996); J. P. Perdew, K.

Burke, M. Ernzerhof, Phys. Rev. Lett. 78 (1997) 1396.

[26] M. Methfessel, A.T. Paxton, Phys. Rev. B 40 (1989) 3616.

[27] P.E. Blöchl, O. Jepsen, O.K. Anderson, Phys. Rev. B 49 (1994) 16223.

[28] H.J. Monkhorst, J.D. Pack, Phys. Rev. B 13 (1976) 5188.

[29] F. Birch, Phys. Rev. 71 (1947) 809.

[30] K.E. Tan, A.P. Horsfield, D.N. Manh, D.G. Pettifor, A.P. Sutton, Phys. Rev. Lett. 76 (1996) 90.

[31] A.L. Bowman, J. Phys. Chem. 65 (1961) 1596. 
[32] A.L. Bowman, Acta. Cryst. 19 (1965) 6.

[33] K. Momma and F. Izumi, J. Appl. Crystallogr. 44 (2011) 1272.

[34] R. Hill, Proc. Phys. Soc. A 65 (1952) 349.

[35] S.P. Dodd, M. Cankurtaran, B. James, J. Mater. Sci. 38 (2003) 1107.

[36] L. López-de-la-Torre, B. Winkler, J. Schreuer, K. Knorr, M. Avalos-Borja, Solid State Comm. 134 (2005) 245.

[37] X.-X. Yu, C.R. Weinberger, G.B. Thompson, Acta Mater. 80 (2014) 341.

[38] G.-H. Lu, M. Huang, M. Cuma, F. Liu, Surf. Sci. 588 (2005) 61.

[39] V. Fiorentini, M. Methfessel, J. Phys. Condens. Matter 8 (1996) 6525.

[40] Y. Han, D.-J. Liu, Phys. Rev. B 80 (2009) 155404.

[41] J.-M. Zhang, F. Ma, K.-W. Xu, Appl. Surf. Sci. 229 (2004) 34.

[42] D.J. Rowcliffe, G. Thomas, Mater. Sci. Eng. 18 (1975) 231. 


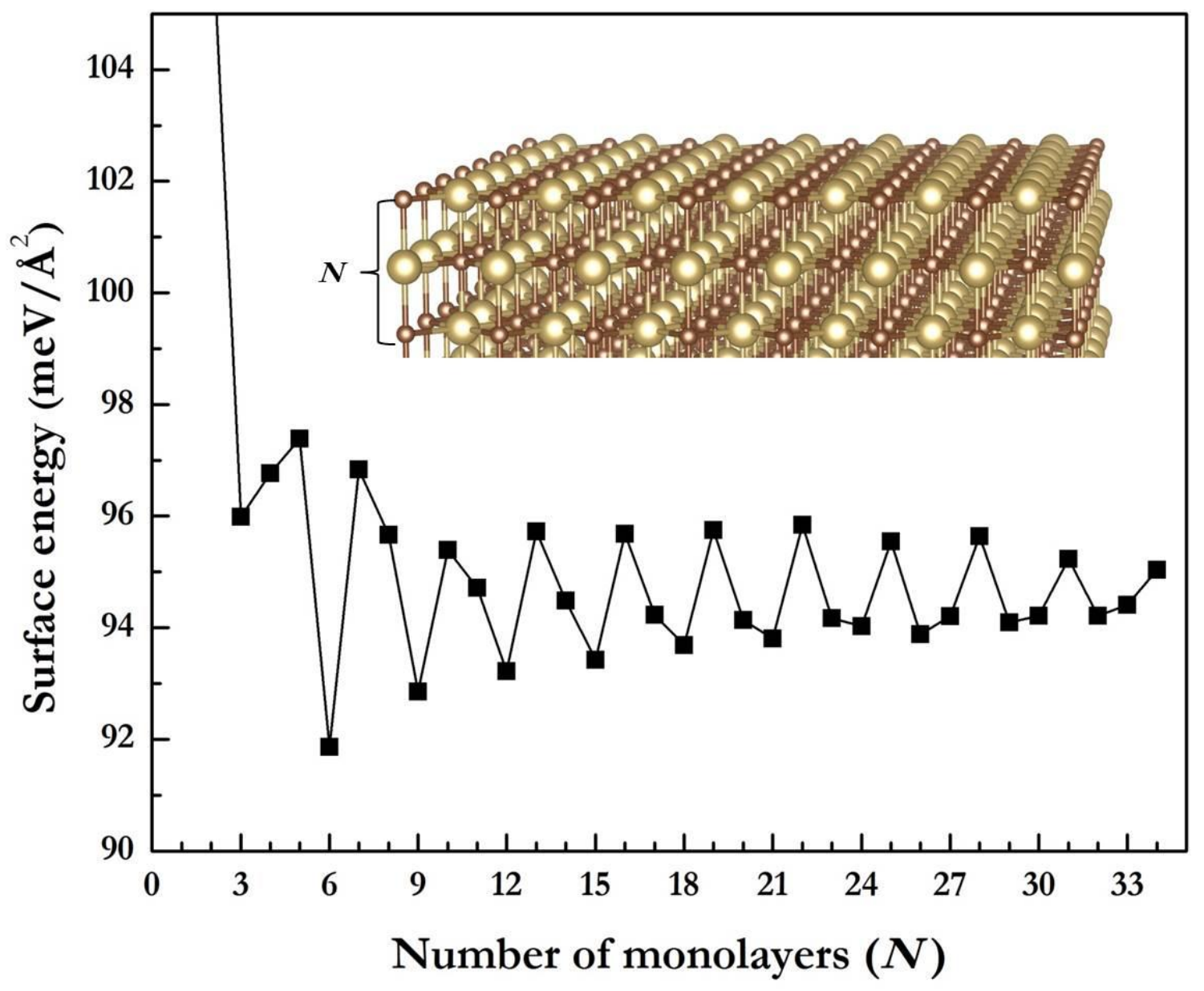

